\title{
D2D-Aided Multi-Antenna Multicasting in a Dense Network
}

\author{
Placido Mursia, ${ }^{1}$ Italo Atzeni, ${ }^{2}$ Mari Kobayashi, ${ }^{3}$ and David Gesbert ${ }^{1}$ \\ ${ }^{1}$ EURECOM, Communication Systems Department, Sophia Antipolis, France \\ ${ }^{2}$ University of Oulu, Centre for Wireless Communications, Oulu, Finland \\ ${ }^{3}$ Technical University of Munich, Munich, Germany \\ Emails: \{placido.mursia, david.gesbert\}@eurecom.fr, italo.atzeni@oulu.fi, mari.kobayashi@tum.de
}

\begin{abstract}
We consider the problem of multicast transmission in cooperative cellular systems, where a base station (BS) aims at conveying a common message to a set of user equipments (UEs) that are also connected with each other via device-todevice (D2D) links. Assuming statistical channel knowledge at the BS, we design a two-phase scheme that exploits multi-antenna transmission at the BS and D2D communications between the UEs to achieve a non-vanishing multicast rate when the number of UEs grows large. This represents a non-trivial extension of our previous work, which is restricted to the case of instantaneous channel knowledge at the BS (and is thus impractical for dense networks). Finally, we study the scaling of the resulting multicast rate as a function of the number of UEs and BS antennas for different practical scenarios.
\end{abstract}

Index Terms-Cooperative communications, device-to-device communications, multicasting, statistical precoding.

\section{INTRODUCTION}

We study the multicast channel, in which a transmitter aims at conveying a common message to a set of receivers. Such a channel is relevant to many emerging applications, ranging from media streaming in wireless edge caching [1], [2] to broadcasting of safety messages in vehicular networks [3]. Multicasting over wireless channels is challenging due to the worst-user-kills-all effect, whereby the multicast capacity vanishes as the number of user equipments (UEs) increases significantly [4], [5].

Cooperative multicasting represents a new transmission paradigm that promises to bring significant performance gains in multicast applications with a large UE population (such as connected car networks) [6], [7]. In this respect, device-todevice (D2D) communications can be used to overcome the vanishing multicast capacity when the number of UEs grows large [8]-[10]. Cooperative multicasting schemes usually divide the total transmission duration in two phases: in the first phase, the BS multicasts a common message to the UEs and, in the second phase, the UEs who have successfully decoded the message in the first phase jointly (yet non-coherently) retransmit it to the rest of the UE population. Such two-phase

The work of P. Mursia is supported by Marie Skłodowska-Curie actions (MSCA-ITN-ETN 722788 SPOTLIGHT). The work of I. Atzeni is supported by the Academy of Finland under grant no. 312648 and 318927 (6Genesis Flagship). The work of M. Kobayashi and D. Gesbert is supported by the French-German Academy towards Industry 4.0 (SeCIF project) under Institut Mines-Telecom. protocols have been extensively studied in the literature for the case of single-antenna BS (e.g., in [8]-[11]) and have been extended to multi-antenna BS in our recent work [12].

In this paper, we propose a two-phase protocol where the precoding optimization at the multi-antenna BS relies only on the channel statistics. This is a key difference with respect to [12], which assumes perfect channel state information (CSI) at the BS. An anticipated advantage of this approach is the great reduction in the overheard necessary to acquire costly instantaneous CSI, which is a major limitation in scenarios with large number of UEs. Hence, we jointly design a simple UE scheduler and the corresponding optimal precoding matrix such that the multicast rate is maximized while ensuring a target successful decoding probability of all the UEs after the two phases. Furthermore, we show that the proposed scheme achieves a non-vanishing multicast rate in the limit of a large number of UEs and/or BS antennas.

\section{SySTEM MODEL}

Consider a wireless network where a BS equipped with $M$ transmit antennas serves a set $\mathcal{K} \triangleq\{1, \ldots, K\}$ of singleantenna UEs uniformly randomly distributed over a finite area, with $K \gg M$. The UEs can communicate with each other via D2D links and operate in half-duplex mode. We focus on a cooperative multicast scheme divided in two phases as follows (see Figure 1): $i$ ) in the first phase, the BS multicasts the transmit signal $\mathbf{x}_{0} \in \mathbb{C}^{M \times 1}$ containing a common message for all the UEs with transmit covariance matrix $\boldsymbol{\Gamma} \triangleq \mathbb{E}\left[\mathbf{x}_{0} \mathbf{x}_{0}^{\mathrm{H}}\right] \in \mathbb{C}^{M \times M}$ (with $\operatorname{tr}(\boldsymbol{\Gamma}) \leq 1$ ) and at rate $r$, referred to as multicast rate; ii) in the second phase, the UEs who have successfully decoded the common message in the first phase jointly (yet non-coherently) retransmit it to the rest of the network through the D2D links.

Let us define the uniform linear array (ULA) response vector at the BS for a given steering angle $\theta_{k}$ as

$$
\mathbf{a}_{k} \triangleq\left[1, e^{j 2 \pi \delta \cos \left(\theta_{k}\right)}, \ldots, e^{j 2 \pi \delta(M-1) \cos \left(\theta_{k}\right)}\right]^{\mathrm{T}} \in \mathbb{C}^{M \times 1}
$$

where $\delta$ is the antenna spacing-wavelength ratio, with $\left\|\mathbf{a}_{k}\right\|^{2}=$ $M$. The channel from the BS to UE $k$ is given by $\mathbf{h}_{k} \triangleq$ $\eta_{k} \sqrt{\gamma_{k}} \mathbf{a}_{k} \in \mathbb{C}^{M \times 1}$, where $\eta_{k} \sim \mathcal{C N}(0,1)$ is the smallscale fading coefficient and $\gamma_{k}$ is the average channel power gain: here, we assume $\gamma_{k}=d_{k}^{-\alpha}$ and $\gamma_{k}=d_{k}^{-\beta}$ in case of 


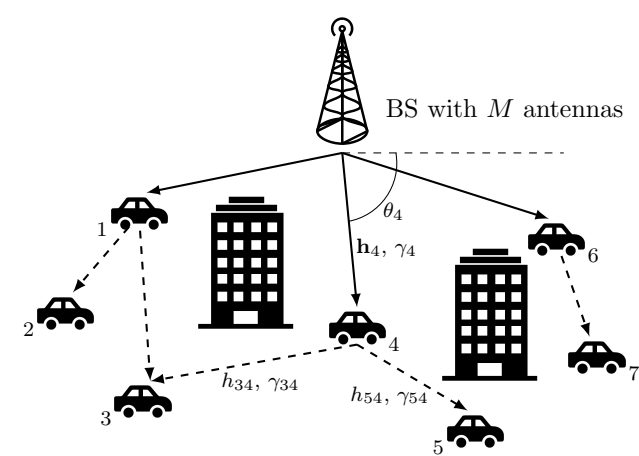

$\rightarrow$ First phase

$\rightarrow$ Second phase

$K$ single-antenna UEs Relay set $\mathcal{U}=\{1,4,6\}$

Fig. 1. A BS equipped with $M$ antennas multicasts a common message to a given subset of UEs with a given precoding strategy in the first phase (solid lines). The UEs who successfully decode the message retransmit it in the second phase to the remaining UEs via D2D links (dashed lines).

line-of-sight (LoS) and non-line-of-sight (NLoS) conditions, respectively, with $2 \leq \alpha<\beta \leq 4$ and where $d_{k}$ denotes the distance between the BS and UE $k$. Hence, the receive signal in the first phase reads as

$$
y_{k, 1} \triangleq \sqrt{p_{0}} \mathbf{h}_{k}^{\mathrm{H}} \mathbf{x}_{0}+n_{k}, \quad \forall k \in \mathcal{K}
$$

where $p_{0}$ denotes the transmit power at the BS and $n_{k} \sim$ $\mathcal{C N}\left(0, \sigma^{2}\right)$ is the noise term at UE $k$. The D2D channel between UEs $k$ and $j$ is denoted by $h_{j k} \triangleq \eta_{j k} \sqrt{\gamma_{j k}} \in \mathbb{C}$, where $\eta_{j k} \sim \mathcal{C N}(0,1)$ is the small-scale fading coefficient and $\gamma_{j k}$ is the average channel power gain (defined similarly to $\gamma_{k}$ for LoS and NLoS conditions between the UEs). The receive signal in the second phase is expressed as

$$
y_{k, 2} \triangleq \sum_{j \in \mathcal{U}} \sqrt{p_{j}} h_{k j} x_{j}+n_{k}, \quad \forall k \in \mathcal{K} \backslash \mathcal{U}
$$

where $\mathcal{U} \subset \mathcal{K}$ is the set of UEs who have successfully decoded the message in the first phase, $p_{j}$ is the transmit power at $\mathrm{UE} j$, and $x_{j}$ is the transmit signal in the second phase (with $\mathbb{E}\left[\left|x_{j}\right|^{2}\right]=1$ ).

\section{D2D-Aided Multi-Antenna Multicasting in A DENSE NETWORK}

In this paper, we assume statistical CSI at the BS, which consists of the position of the UEs within its coverage area together with a map of the latter. Building on this information, the BS can easily obtain the average channel gains $\left\{\gamma_{k}\right\}_{k \in \mathcal{K}}$ and $\left\{\gamma_{k j}\right\}_{k, j \in \mathcal{K}}$ together with the steering angles $\left\{\theta_{k}\right\}_{k \in \mathcal{K}}$. On the other hand, we assume that the BS has no knowledge of the small-scale fading coefficients $\left\{\eta_{k}\right\}_{k \in \mathcal{K}}$ and $\left\{\eta_{k j}\right\}_{k, j \in \mathcal{K}}$.

The BS multicasts the common message at rate $r \triangleq$ $\log _{2}(1+s)$, where $s$ is a design parameter to be optimized. Hence, each UE $k$ successfully decodes in the first phase if its receive signal-to-noise ratio (SNR) is greater than $s$, which occurs with probability

$$
\begin{aligned}
\mathrm{P}_{k, 1}(s, \boldsymbol{\Gamma}) & \triangleq \mathbb{P}\left[\rho_{0}\left|\eta_{k}\right|^{2} \gamma_{k} \mathbf{a}_{k}^{\mathrm{H}} \boldsymbol{\Gamma} \mathbf{a}_{k} \geq s\right] \\
& =\exp \left(-\frac{s}{\rho_{0} \gamma_{k} \mathbf{a}_{k}^{\mathrm{H}} \boldsymbol{\Gamma} \mathbf{a}_{k}}\right)
\end{aligned}
$$

where $\rho_{0} \triangleq p_{0} / \sigma^{2}$ denotes the transmit SNR at the BS. Let $z_{k}(s, \boldsymbol{\Gamma}) \triangleq \mathbb{1}\left[\rho_{0}\left|\eta_{k}\right|^{2} \gamma_{k} \mathbf{a}_{k}^{\mathrm{H}} \boldsymbol{\Gamma} \mathbf{a}_{k} \geq s\right]$ be a binary variable equal to one if UE $k$ successfully decodes the message in the first phase and to zero otherwise. We use $\mathrm{P}_{2}(s, \boldsymbol{\Gamma})$ to denote the joint success probability, i.e., the probability that all UEs successfully decode the message after the two phases, defined as

$\mathrm{P}_{2}(s, \boldsymbol{\Gamma}) \triangleq \mathbb{E}\left[\prod_{k \in \mathcal{K}} \mathbb{P}\left[\left|\sum_{j \neq k} h_{k j} \rho_{j}\right|^{2} \geq s\left(1-z_{k}(s, \boldsymbol{\Gamma})\right) \mid \mathbf{z}(s, \boldsymbol{\Gamma})\right]\right]$

where $\rho_{j} \triangleq p_{j} / \sigma^{2}$ is the transmit SNR at $\operatorname{UE} j$ and $\mathbf{z}(s, \boldsymbol{\Gamma}) \triangleq$ $\left[z_{1}(s, \boldsymbol{\Gamma}), \ldots, z_{K}(s, \boldsymbol{\Gamma})\right] \in\{0,1\}^{K}$. Building on [10, Th. 4], a deterministic-equivalent expression for $\mathrm{P}_{2}(s, \boldsymbol{\Gamma})$ is derived in the following proposition.

Proposition 1. Assuming independent channels between the $B S$ and the UEs and between UEs, we have

$$
\mathrm{P}_{2}(s, \boldsymbol{\Gamma}) \underset{K \rightarrow \infty}{\stackrel{\mathbb{P}}{\rightarrow}} \overline{\mathrm{P}}_{2}(s, \boldsymbol{\Gamma})
$$

where

$$
\overline{\mathrm{P}}_{2}(s, \boldsymbol{\Gamma}) \triangleq \exp \left(-\sum_{k \in \mathcal{K}} \frac{s\left(1-\mathrm{P}_{k, 1}(s, \boldsymbol{\Gamma})\right)}{\sum_{j \in \mathcal{K} \backslash\{k\}} \mathrm{P}_{j, 1}(s, \boldsymbol{\Gamma}) \gamma_{j k} \rho_{j}}\right)
$$

is the deterministic equivalent of $\mathrm{P}_{2}(s \boldsymbol{\Gamma})$.

\section{A. Problem Formulation}

We consider the problem of maximizing the outage multicast rate, i.e., the multicast rate subject to an outage constraint on the joint success probability, in a dense network where the number of UE is increased within a finite network area. Assuming that the total transmission time is divided equally between the two phases, we study the optimization problem

$$
\begin{array}{cl}
\max _{s>0, \boldsymbol{\Gamma} \succeq \mathbf{0}} & \log _{2}(1+s) \\
\text { s.t. } & \operatorname{tr}(\boldsymbol{\Gamma}) \leq 1 \\
& \overline{\mathrm{P}}_{2}(s, \overline{\boldsymbol{\Gamma}}) \geq 1-\epsilon
\end{array}
$$

where $\epsilon \in[0,1)$ is the target outage probability after the two phases and where we have replaced $\mathrm{P}_{2}(s, \boldsymbol{\Gamma})$ with its deterministic equivalent defined in Proposition 1.

\section{B. Statistical Multi-Antenna Multicasting}

In absence of the second phase of cooperative D2D communications, the objective of the BS is to maximize the multicast rate $r_{1} \triangleq \log _{2}\left(1+s_{1}\right)$ subject to an outage constraint on the joint success probability over the first phase, i.e., $\overline{\mathrm{P}}_{1}\left(s_{1}, \boldsymbol{\Gamma}\right) \geq$ $1-\epsilon$, where we have defined $\overline{\mathrm{P}}_{1}\left(s_{1}, \boldsymbol{\Gamma}\right) \triangleq \prod_{k \in \mathcal{K}} \mathrm{P}_{k, 1}\left(s_{1}, \boldsymbol{\Gamma}\right)$. In this context, for a given transmit covariance matrix $\Gamma$, the outage multicast rate $r_{1}$ is maximized when the outage constraint is satisfied with equality, leading to

$$
s_{1}=\rho_{0} \log \left(\frac{1}{1-\epsilon}\right)\left(\sum_{k \in \mathcal{K}} \frac{1}{\gamma_{k} \mathbf{a}_{k}^{\mathrm{H}} \boldsymbol{\Gamma} \mathbf{a}_{k}}\right)^{-1} .
$$

Then, the optimal transmit covariance is obtained by solving

$$
\begin{array}{ll}
\min _{\boldsymbol{\Gamma} \succeq \mathbf{0}} & \sum_{k \in \mathcal{K}} \frac{1}{\gamma_{k} \mathbf{a}_{k}^{\mathrm{H}} \boldsymbol{\Gamma} \mathbf{a}_{k}} \\
\text { s.t. } & \operatorname{tr}(\boldsymbol{\Gamma}) \leq 1
\end{array}
$$


by means of semidefinite programming. This case is referred to in the following as statistical multi-antenna multicasting (SMAM).

Proposition 2. Assume that the set $\mathcal{K}$ consists of $M U E s$ exhibiting mutually orthogonal array responses, i.e.,

$$
\sum_{k \in \mathcal{K}} \mathbf{a}_{k} \mathbf{a}_{k}^{\mathrm{H}}=M \mathbf{I}_{M}
$$

with $\mathbf{I}_{M}$ being the $M$-dimensional identity matrix. Then, the optimal transmit covariance matrix for problem (11) can be written in closed form as

$$
\boldsymbol{\Gamma}_{1} \triangleq \frac{1}{M \nu_{\mathcal{K}}} \sum_{k \in \mathcal{K}} \frac{1}{\sqrt{\gamma_{k}}} \mathbf{a}_{k} \mathbf{a}_{k}^{\mathrm{H}}
$$

with $\nu_{\mathcal{K}} \triangleq \sum_{k \in \mathcal{K}} 1 / \sqrt{\gamma_{k}}$.

Proof. See Appendix A.

Note that a set of array response vectors satisfying (12) can be obtained as the columns of the $M$-dimensional discrete Fourier transform (DFT) matrix or, alternatively, it can be constructed along specific virtual angles as described in [13].

\section{D2D-Aided Statistical Multi-Antenna Multicasting}

In presence of the second phase of cooperative D2D communications, solving problem (9) is non-trivial as $\overline{\mathrm{P}}_{2}(s, \boldsymbol{\Gamma})$ in (8) is non-convex in both the optimization variables. To reduce the complexity, we decouple the optimization of $\boldsymbol{\Gamma}$ and $s$ as follows. Assuming that the UE distribution is uniform in the angular domain, we build on Proposition 2 and construct the subset $\mathcal{U}$ of UEs to be targeted in the first phase by selecting $M$ UEs satisfying the condition in (12): by doing so, the BS spreads its transmit power along a set of directions that span the whole angular domain. ${ }^{1}$ In this setting, the transmit covariance matrix that maximizes the outage multicast rate over the UEs in $\mathcal{U}$ is given by $\boldsymbol{\Gamma}_{1}$ in (13), and we define the resulting outage multicast rate is $r=\log _{2}(1+s)$ with (cf. (10))

$$
s=\rho_{0} \log \left(\frac{1}{1-\epsilon_{1}}\right) \frac{M}{\nu_{\mathcal{U}}^{2}}
$$

where we have defined $\nu_{\mathcal{U}} \triangleq \sum_{k \in \mathcal{U}} 1 / \sqrt{\gamma_{k}}$ and where $\epsilon_{1} \in$ $[0,1)$ denotes the target outage probability after the first phase over the UEs in $\mathcal{U}$. Next, we optimize the value of $\epsilon_{1}$ in order to maximize the multicast rate with target outage probability $\epsilon$ after the two phases. The target outage probabilities $\epsilon_{1}$ and $\epsilon$ (i.e., after the first and the second phase, respectively) are related through (14) as

$$
\sum_{k \in \mathcal{K}} \frac{s\left(1-\exp \left(-\frac{s}{\rho_{0} \gamma_{k} \mathbf{a}_{k}^{\mathrm{H}} \boldsymbol{\Gamma} \mathbf{a}_{k}}\right)\right)}{\sum_{j \in \mathcal{K} \backslash\{k\}} \exp \left(-\frac{s}{\rho_{0} \gamma_{j} \mathbf{a}_{j}^{\mathrm{H}} \boldsymbol{\Gamma} \mathbf{a}_{j}}\right) \gamma_{j k} \rho_{j}}=\log \left(\frac{1}{1-\epsilon}\right)
$$

\footnotetext{
${ }^{1}$ As $K$ is large, we assume that it is always possible to select $M$ UEs whose steering angles satisfy (12).
}

(with $s$ being a function of $\epsilon_{1}$ ), which is simply obtained by plugging (5) into (8) and equating it to the target success probability $1-\epsilon$. This case is referred to in the following as D2D-aided statistical multi-antenna multicasting (D2DSMAM). Note that, as the left-hand-side of (15) is a monotonically decreasing function of $\epsilon_{1}$, the latter can be optimized efficiently via bisection methods.

\section{Asymptotic behavior of D2D-SMAM}

In this section, we study the asymptotic behavior of the proposed D2D-SMAM scheme as a function of the number of UEs and BS antennas. Considering the expression in (15) when $\epsilon \rightarrow 0$ (and, hence, $\epsilon_{1} \rightarrow 0$ ), we have $\exp \left(-s /\left(\rho_{0} \gamma_{k} \mathbf{a}_{k}^{\mathrm{H}} \boldsymbol{\Gamma} \mathbf{a}_{k}\right)\right) \simeq 1-s /\left(\rho_{0} \gamma_{k} \mathbf{a}_{k}^{\mathrm{H}} \boldsymbol{\Gamma} \mathbf{a}_{k}\right)$ and, thus, we can write the following asymptotic approximation of $s$ :

$$
s \underset{\epsilon \rightarrow 0}{\simeq} \bar{s} \triangleq \sqrt{\frac{\rho_{0} \log \left(\frac{1}{1-\epsilon}\right)}{\sum_{k \in \mathcal{K}} \frac{1}{\gamma_{k} \mathbf{a}_{k}^{\mathrm{H}} \mathbf{\Gamma} \mathbf{a}_{k}}\left(\sum_{j \in \mathcal{K} \backslash\{k\}} \gamma_{j k} \rho_{j}\right)^{-1}}} .
$$

We assume that $d_{k} \in\left[R_{\min }, R_{\max }\right] \forall k$, where $R_{\min }$ and $R_{\max }$ denote the minimum and maximum distance from UE $k$ to the $\mathrm{BS}$ respectively. Hence, let us consider the worst-case scenario where

$$
\begin{aligned}
\gamma_{k} & =R_{\text {max }}^{-\beta}, \quad \forall k \in \mathcal{K} \\
\gamma_{j k} & =\left(2 R_{\max }\right)^{-\beta}, \quad \forall k, j \in \mathcal{K} .
\end{aligned}
$$

In this setting, we have

$$
\begin{aligned}
\mathbf{a}_{k}^{\mathrm{H}} \boldsymbol{\Gamma} \mathbf{a}_{k} & =\frac{1}{M \nu \mathcal{U}} \sum_{j \in \mathcal{U}} \frac{1}{\sqrt{\gamma_{j}}}\left|\mathbf{a}_{k}^{\mathrm{H}} \mathbf{a}_{j}\right|^{2} \\
& \geq \frac{M}{\nu_{\mathcal{U}}} R_{\min }^{\alpha / 2}
\end{aligned}
$$

where (20) follows from restricting the summation in (19) to the worst case in which $\left|\mathbf{a}_{k}^{\mathrm{H}} \mathbf{a}_{j}\right|^{2}>0$ only for the two indices $j \in \mathcal{U}$ corresponding to the adjacent steering angles with respect to UE $k$. In the following, we consider the case in which both the number of UEs $K$ and the number of BS antennas $M$ increase with fixed ratio $c \triangleq K / M>1$, as well as the case in which $K$ increases for a fixed $M$. Finally, we can lower bound $\bar{s}$ in (16) as

$$
\bar{s} \underset{K \rightarrow \infty}{ } \begin{cases}\sqrt{\frac{\rho_{0} \rho_{\mathrm{UE}} \log \left(\frac{1}{1-\epsilon}\right) R_{\mathrm{min}}^{\alpha / 2}}{2^{\beta} \nu_{\mathcal{U}} R_{\max }^{2 \beta}(c-1)} K}, & \text { for } c=\frac{K}{M}>1 \\ \sqrt{\frac{\rho_{0} \rho_{\mathrm{UE}} \log \left(\frac{1}{1-\epsilon}\right) R_{\min }^{\alpha / 2}}{2^{\beta} \nu_{\mathcal{U}} R_{\max }^{2 \beta}} M,} & \text { for fixed } M .\end{cases}
$$

Based on (21), we obtain a lower bound on the outage multicast rate that increases as $\log _{2}(1+\mathcal{O}(\sqrt{K}))$ in the first case and that is constant (yet non-vanishing) in the second case.

\section{Numerical Results AND Discussion}

In this section, we compare the asymptotic expressions obtained in Section III-D with numerical simulations. In doing so, we assume that the links between the BS and the UEs and 


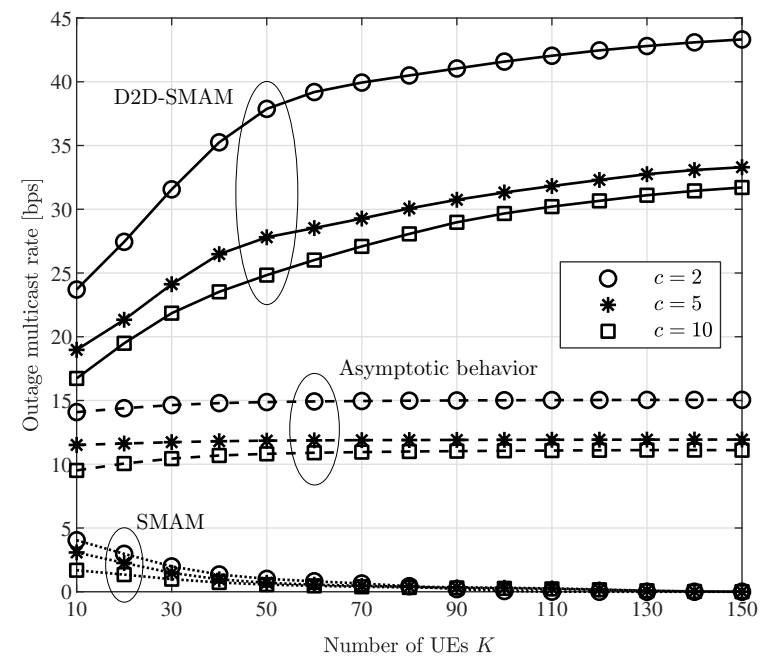

Fig. 2. Outage multicast rate obtained with the SMAM and D2D-SMAM algorithms, together with the asymptotic behavior of latter, versus the number of UEs for different values of $c=K / M$. As expected, the D2D-SMAM algorithm achieves a non-vanishing multicast rate as opposed to the SMAM algorithm, whereas the asymptotic behavior increases with $K$.

the D2D links between UEs are in LoS or NLoS conditions with equal probability. We build the set $\mathcal{U}$ by identifying $M$ UEs whose steering angles satisfy the condition in (12), out of which $|\mathcal{U}| / 4$ are assumed to be in NLoS conditions with respect to the BS. The target outage probability is fixed to $\epsilon=10^{-3}$ and we consider a total transmission bandwidth of $10 \mathrm{MHz}$. For simplicity, we assume that all the UEs have the same transmit SNR, i.e., $\left\{\rho_{k}=\rho_{\mathrm{UE}}\right\}_{k \in \mathcal{K}}$, and set $\rho_{0}=20 \mathrm{~dB}$ and $\rho_{\mathrm{UE}}=10 \mathrm{~dB}$. Furthermore, we fix the LoS and NLoS pathloss exponents to $\alpha=2$ and $\beta=4$, respectively. Lastly, the numerical results are averaged over $3 \times 10^{3}$ uniformly random UE locations within a half-disc area with minimum radius $R_{\min }=10 \mathrm{~m}$ and maximum radius $R_{\max }=100 \mathrm{~m}$ from the BS.

Figure 2 shows the outage multicast rate for the proposed D2D-SMAM and SMAM algorithms together with the asymptotic behavior in (21) when both the number of UEs $K$ and the number of BS antennas $M$ increase with fixed ratio $c=K / M$. Unlike the baseline SMAM algorithm, the proposed D2DSMAM algorithm achieves an increasing outage multicast rate as the number of UEs grows large, even when the target outage probability is small as in the considered scenario. Recall that this is achieved on the basis of statistical CSI only (and, thus, with minimal overhead). Such benefits stem mainly from efficient use of the resources available at both the BS and the UEs to reach the whole UE population, as opposed to the baseline scheme where the BS alone does not have enough spatial degrees of freedom. In addition, the asymptotic behavior of the D2D-SMAM algorithm increases as $\log _{2}(1+\mathcal{O}(\sqrt{K}))$, although with slow pace due to the pessimistic assumptions made in (17)-(18). By fixing the number of UEs $K$, an increase in the number of BS antennas $M$ brings substantial performance gains, as shown in Figure 3. Here, the outage multicast rate achieve by the D2D-SMAM algorithm is again increasing with $K$, whereas the asymptotic behavior in (21)

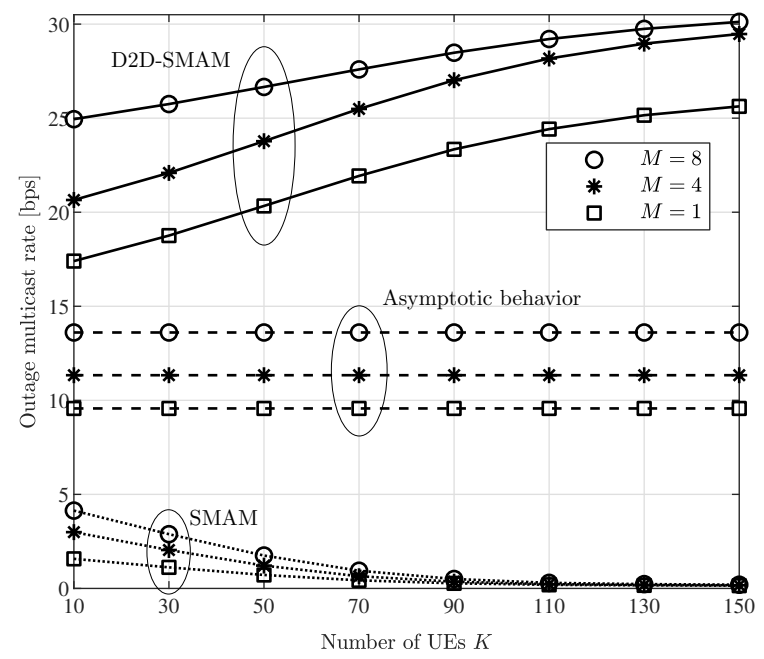

Fig. 3. Outage multicast rate obtained with the SMAM and D2D-SMAM algorithms, together with the asymptotic behavior of the latter, versus the number of UEs for different values of $M$. As expected, the D2D-SMAM algorithm achieves a non-vanishing multicast rate as opposed to the SMAM algorithm, whereas the asymptotic behavior is constant with $K$.

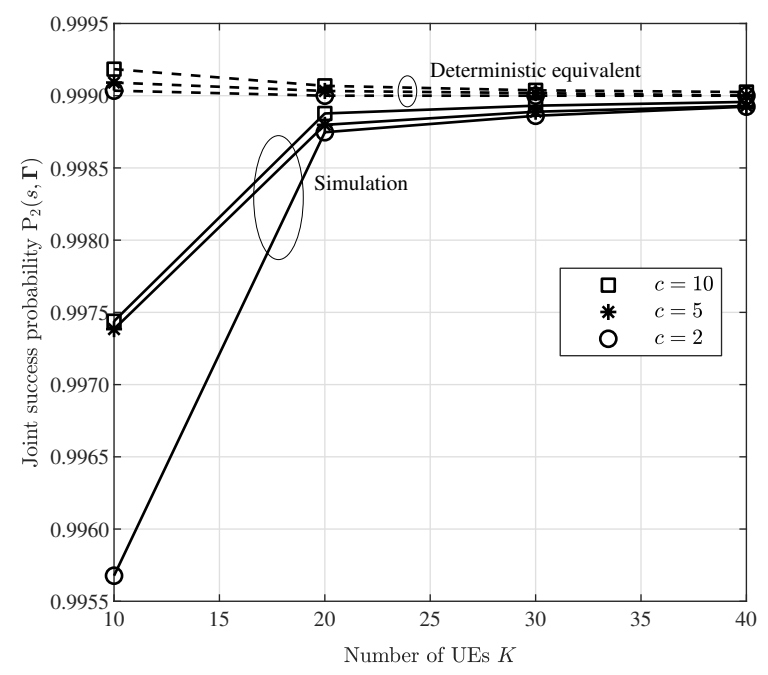

Fig. 4. Joint success probability over the two phases in (6) and its deterministic equivalent in (8) versus the number of UEs for different values of $c=K / M$. The asymptotic behavior of the joint success probability is accurate even for small values of $K$.

is constant with $K$ (i.e., non-vanishing). An increase in $M$ is also beneficial for the SMAM algorithm, although the obtained outage multicast rate still vanishes with $K$. Lastly, Figure 4 compares the joint success probability obtained via numerical simulations with its deterministic equivalent $\overline{\mathrm{P}}_{2}(s, \boldsymbol{\Gamma})$ in (8) when both the number of UEs $K$ and the number of BS antennas $M$ increase with fixed ratio $c=K / M$. Remarkably, $\overline{\mathrm{P}}_{2}(s, \boldsymbol{\Gamma})$ is accurate even for small values of $K$.

\section{CONCLusions}

We propose a two-phase multicasting scheme that exploits both multi-antenna transmission through precoding at the BS and cooperative D2D communications among UEs. Unlike our previous work [12], our scheme is able to achieve a 
non vanishing outage multicast rate on the basis of statistical CSI only (and, thus, with minimal overhead). Our asymptotic analysis shows that the resulting outage multicast rate grows with the number of UEs $K$ when the latter is increased together with the number of BS antennas $M$ with a fixed ratio, whereas it achieves a constant (non-vanishing) outage multicast rate when $K$ is increased for a fixed $M$.

\section{APPENDIX A}

\section{PROOF OF PROPOSITION 2}

Since problem (11) is convex, a given $\Gamma$ is optimal if and only if it satisfies the Karush-Kuhn-Tucker (KKT) conditions. Let us define the Lagrangian and its gradient as

$$
\begin{aligned}
\mathcal{L}(\boldsymbol{\Gamma}, \mu, \boldsymbol{\Psi}) & \triangleq \sum_{k \in \mathcal{K}} \frac{1}{\gamma_{k} \mathbf{a}_{k}^{\mathrm{H}} \boldsymbol{\Gamma} \mathbf{a}_{k}}+\mu(\operatorname{tr}(\boldsymbol{\Gamma})-1)-\operatorname{tr}(\boldsymbol{\Psi} \boldsymbol{\Gamma}), \\
\nabla \mathcal{L}(\boldsymbol{\Gamma}, \mu, \boldsymbol{\Psi}) & =-\sum_{k \in \mathcal{K}} \frac{1}{\gamma_{k}\left(\mathbf{a}_{k}^{\mathrm{H}} \boldsymbol{\Gamma} \mathbf{a}_{k}\right)^{2}} \mathbf{a}_{k} \mathbf{a}_{k}^{\mathrm{H}}+\mu \mathbf{I}_{M}-\boldsymbol{\Psi}
\end{aligned}
$$

respectively, where we have introduced the dual variables $\mu \in$ $\mathbb{R}$ and $\Psi \in \mathbb{C}^{M \times M}$. The KKT conditions of problem (11) can be written as

$$
\begin{aligned}
& \sum_{k \in \mathcal{K}} \frac{1}{\gamma_{k}\left(\mathbf{a}_{k}^{\mathrm{H}} \boldsymbol{\Gamma} \mathbf{a}_{k}\right)^{2}} \mathbf{a}_{k} \mathbf{a}_{k}^{\mathrm{H}}=\mu \mathbf{I}_{M}-\mathbf{\Psi}, \\
& \operatorname{tr}(\boldsymbol{\Gamma}) \leq 1, \boldsymbol{\Gamma} \succeq \mathbf{0}, \\
& \mu \geq 0, \boldsymbol{\Psi} \succeq \mathbf{0}, \\
& \mu(\operatorname{tr}(\boldsymbol{\Gamma})-1)=0, \quad \boldsymbol{\Psi} \boldsymbol{\Gamma}=\mathbf{0} .
\end{aligned}
$$

The condition in (24a) suggests that the transmit covariance matrix has the structure

$$
\boldsymbol{\Gamma}=\sum_{k \in \mathcal{K}} w_{k} \mathbf{a}_{k} \mathbf{a}_{k}^{\mathrm{H}}
$$

where $\sum_{k \in \mathcal{K}} w_{k}=1 / M$ implies $\operatorname{tr}(\boldsymbol{\Gamma})=1$ and $\left\{w_{k} \geq 0\right\}_{k \in \mathcal{K}}$ implies $\boldsymbol{\Gamma} \succeq \mathbf{0}$. From (25), we can write

$$
\mathbf{a}_{k}^{\mathrm{H}} \boldsymbol{\Gamma} \mathbf{a}_{k}=\sum_{j \in \mathcal{K}} w_{j} \phi_{k j}
$$

where we have defined $\phi_{k j} \triangleq\left|\mathbf{a}_{k}^{\mathrm{H}} \mathbf{a}_{j}\right|^{2}$, with $\boldsymbol{\Phi} \triangleq\left[\phi_{k j}\right]_{k, j \in \mathcal{K}} \in$ $\mathbb{C}^{K \times K}$ symmetric with diagonal elements equal to $M^{2}$. Plugging (25) into (24), we can rewrite the KKT conditions as

$$
\begin{aligned}
& \sum_{k \in \mathcal{K}} \frac{1}{\gamma_{k}\left(\sum_{j \in \mathcal{K}} w_{j} \phi_{k j}\right)^{2}} \mathbf{a}_{k} \mathbf{a}_{k}^{\mathrm{H}}=\mu \mathbf{I}_{M}-\mathbf{\Psi}, \\
& \sum_{k \in \mathcal{K}} w_{k}=\frac{1}{M},\left\{w_{k} \geq 0\right\}_{k \in \mathcal{K}}, \\
& \mu \geq 0, \mathbf{\Psi} \succeq \mathbf{0}, \\
& \mu\left(\sum_{k \in \mathcal{K}} w_{k}-\frac{1}{M}\right)=0, \quad \boldsymbol{\Psi} \sum_{k} w_{k} \mathbf{a}_{k} \mathbf{a}_{k}^{\mathrm{H}}=\mathbf{0} .
\end{aligned}
$$

Let us define $\mathbf{w} \triangleq\left[w_{1}, \ldots, w_{K}\right]^{\mathrm{T}} \in \mathbb{R}^{K \times 1}$ and let $\mathbf{e}_{k}$ denote the $k$ th column of $\mathbf{I}_{K}$. Choosing the weights that satisfy (27b) allows us to set $\boldsymbol{\Psi}=\mathbf{0}$ and, from (27a), we can show that

$$
\mathbf{w}=\frac{1}{\sqrt{\mu M}} \boldsymbol{\Phi}^{-1} \mathbf{b}
$$

where we have defined

$$
\mathbf{b} \triangleq\left[\frac{1}{\sqrt{\gamma_{1} \mathbf{1}^{\mathrm{T}} \boldsymbol{\Phi}^{-1} \mathbf{e}_{1}}}, \ldots, \frac{1}{\sqrt{\gamma_{K} \mathbf{1}^{\mathrm{T}} \boldsymbol{\Phi}^{-1} \mathbf{e}_{K}}}\right]^{\mathrm{T}} .
$$

On the other hand, the dual variable $\mu$ can be obtained by plugging (28) into the first condition in $(27 \mathrm{~d})$, i.e.,

$$
\mu=M\left(\mathbf{1}^{\mathrm{T}} \boldsymbol{\Phi}^{-1} \mathbf{b}\right)^{2}
$$

and, by plugging (30) into (28), we obtain

$$
w_{k}=\frac{\mathbf{e}_{k}^{\mathrm{T}} \boldsymbol{\Phi}^{-1} \mathbf{b}}{M \mathbf{1}^{\mathrm{T}} \boldsymbol{\Phi}^{-1} \mathbf{b}}, \quad \forall k \in \mathcal{K} .
$$

Finally, choosing $\left\{w_{k}\right\}_{k \in \mathcal{K}}$ as in (31), $\mu$ as in (30), and $\boldsymbol{\Psi}=\mathbf{0}$ readily satisfies $(27 b)-(27 d)$, whereas $(27 a)$ yields

$$
\sum_{k \in \mathcal{K}}\left(\mathbf{1}^{\mathrm{T}} \boldsymbol{\Phi}^{-1} \mathbf{e}_{k}\right) \mathbf{a}_{k} \mathbf{a}_{k}^{\mathrm{H}}=\frac{1}{M} \mathbf{I}_{M}
$$

The latter is satisfied when $\mathbf{\Phi}=M^{2} \mathbf{I}_{K}$, i.e., when $K=M$ and the steering angles of the UEs are such that $\mathbf{a}_{k}^{\mathrm{H}} \mathbf{a}_{j}=$ $0, \forall k \neq j$ (see, e.g., [13] for more details). In this setting, it follows from (31) that $w_{k}=1 /\left(M \sqrt{\gamma_{k}} \nu_{\mathcal{K}}\right)$, from which we obtain the expression of the optimal transmit covariance matrix in (13).

\section{REFERENCES}

[1] M. A. Maddah-Ali and U. Niesen, "Fundamental limits of caching," IEEE Trans. Inf. Theory, vol. 60, no. 5, pp. 2856-2867, May 2014

[2] G. Paschos, E. Bastug, I. Land, G. Caire, and M. Debbah, "Wireless caching: Technical misconceptions and business barriers," IEEE Commun. Mag., vol. 54, no. 8, pp. 16-22, Aug. 2016.

[3] G. Araniti, C. Campolo, M. Condoluci, A. Iera, and A. Molinaro, "LTE for vehicular networking: A survey," IEEE Commun. Mag., vol. 51, no. 5, pp. 148-157, May 2013.

[4] N. Jindal and Z.-Q. Luo, "Capacity limits of multiple antenna multicast," in Proc. IEEE Int. Symp. Inf. Theory (ISIT), Barcelona, Spain, July 2006.

[5] N. D. Sidiropoulos, T. N. Davidson, and Z.-Q. Luo, "Transmit beamforming for physical-layer multicasting," IEEE Trans. Signal Process., vol. 54, no. 6, pp. 2239-2251, June 2006.

[6] A. Asadi, Q. Wang, and V. Mancuso, "A survey on device-to-device communication in cellular networks," IEEE Commun. Surveys Tutorials, vol. 16, no. 4, pp. 1801-1819, 4th quarter 2014.

[7] M. N. Tehrani, M. Uysal, and H. Yanikomeroglu, "Device-to-device communication in $5 \mathrm{G}$ cellular networks: Challenges, solutions, and future directions," IEEE Commun. Mag., vol. 52, no. 5, pp. 86-92, May 2014.

[8] A. Khisti, U. Erez, and G. W. Wornell, "Fundamental limits and scaling behavior of cooperative multicasting in wireless networks," IEEE Trans. Inf. Theory, vol. 52, no. 6, pp. 2762-2770, June 2006.

[9] B. Sirkeci-Mergen and M. C. Gastpar, "On the broadcast capacity of wireless networks with cooperative relays," IEEE Trans. Inf. Theory, vol. 56, no. 8, pp. 3847-3861, Aug. 2010.

[10] T. V. Santana, R. Combes, and M. Kobayashi, "Performance analysis of device-to-device aided multicasting in general network topologies," IEEE Trans. Commun., vol. PP, no. 99, pp. 1-1, 2019.

[11] F. Hou, L. X. Cai, P. Ho, X. Shen, and J. Zhang, "A cooperative multicast scheduling scheme for multimedia services in IEEE 802.16 networks," IEEE Trans. Wireless Commun., vol. 8, no. 3, pp. 1508-1519, Mar. 2009.

[12] P. Mursia, I. Atzeni, D. Gesbert, and M. Kobayashi, "D2D-aided multi-antenna multicasting," in Proc. IEEE Int. Conf. Commun. (ICC), Shanghai, China, May 2019.

[13] A. M. Sayeed, "Deconstructing multiantenna fading channels," IEEE Trans. Signal Process., vol. 50, no. 10, pp. 2563-2579, Oct. 2002. 